\title{
A 45-CM MONSTER
}

\author{
M. A. Schwartz ${ }^{1}$, K. Macedo ${ }^{1}$, A. Zurita ${ }^{1}$, A. Boette ${ }^{2}$, A. Pardini ${ }^{3}$, E. Vilche ${ }^{1}$, C. Winschu ${ }^{1}$, J. Capobianco $^{1}$, \\ L. la Malfa ${ }^{1}$, G. Rodríguez ${ }^{1}$, E. García ${ }^{1}$, G. Folatelli ${ }^{1,5}$, J. Carpinetti ${ }^{1}$, R. Vázquez ${ }^{1,6}$, A. Cruzado ${ }^{1,6}$, \\ and M. Orellana ${ }^{4,5}$
}

\begin{abstract}
In June 2014, the Faculty of Astronomical and Geophysical Sciences of the National University of La Plata, Argentina, received a telescope as a donation from the family of Dr. Rafael Montemayor. This instrument was a personal project of the professor and researcher of the Balseiro Institute (Bariloche, Argentina), in which he worked during the last 17 years of his life, completing $80 \%$ of the mechanical aspects. The mount with a C-ring type design is very robust, the telescope has a parabolic primary mirror $(f=2027 \mathrm{~mm}$ and $D=457.2 \mathrm{~mm})$ prepared to operate in Newtonian focus. Our goal is to complete the assembly and automation of the telescope, which will be used remotely for scientific, educational and outreach purposes. The development of the pointing electronics and control software are complete and have been tested with Meade telescopes.
\end{abstract}

\section{RESUMEN}

En junio de 2014, la Facultad de Ciencias Astronómicas y Geofísicas de la Universidad Nacional de La Plata, Argentina, recibió un telescopio en concepto de donación por parte de la familia del Dr. Rafael Montemayor. Este instrumento fue un proyecto personal del profesor e investigador del Instituto Balseiro (Bariloche, Argentina) en el que estuvo trabajando durante los últimos 17 años de su vida, completando un $80 \%$ de los aspectos mecánicos del telescopio. La montura con un diseño del tipo C-anillo es muy robusta, el telescopio cuenta con un espejo primario parabólico $(f=2027 \mathrm{~mm}$ y $D=457.2 \mathrm{~mm}$ ) preparado para operar en foco newtoniano. Nuestro objetivo es terminar con el armado y automatización del telescopio para que pueda ser usado en forma remota con fines científicos, educativos y de divulgación. El desarrollo de la electrónica de apuntamiento y el software de control están completos y han sido probados con telescopios Meade más pequeños.

Key Words: telescopes

\section{THE MONTEMAYOR LEGACY}

For 17 years, Rafael Montemayor, engineer and $\mathrm{PhD}$ in Physics, dedicated his free time to design and build a telescope aiming to use it for making the best possible images of the night sky of Bariloche, in the province of Río Negro, Argentina. The result of those years of dedication and effort was a great precision mechanical work that he could not complete because of a terminal illness. Rafael's family decided to fulfill his wish and donated the telescope

\footnotetext{
${ }^{1}$ Facultad de Ciencias Astronómicas y Geofísicas, Universidad Nacional de La Plata (UNLP), Paseo del Bosque s/n, B1900 FWA La Plata, Argentina (mschwartz@carina.fcaglp.unlp.edu.ar).

${ }^{2}$ Facultad de Informática, UNLP, Calles 50 y 120, La Plata, Argentina.

${ }^{3}$ Facultad de Ingeniería, UNLP, Calles 1 y 47, B1900 TAG La Plata, Argentina.

${ }^{4}$ Sede Andina, Universidad Nacional de Río Negro, Mitre 630 (8400) Bariloche, Argentina.

${ }^{5}$ Member of the Carrera del Investigador Científico y Tecnológico, CONICET, Argentina.

${ }^{6}$ Instituto de Astrofísica de La Plata (CCT La Plata CONICET, UNLP), Paseo del Bosque s/n, B1900 FWA La Plata, Argentina.
}

to the Faculty of Astronomical and Geophysical Sciences of the National University of La Plata in April 2014. The Faculty took the initiative to complete the construction and automation of the telescope to be used for scientific, teaching and outreach purposes. The Rafael Montemayor Telescope (RMT) had its first light on August 26, 2017. Its automation will be completed by mid 2018. After that, the use of the RMT will be tested for a period of three months on the campus of the Faculty in La Plata before changing facilities to a site of good seeing conditions for astronomical observation (see Figure 1).

\section{CONSTRUCTION AND AUTOMATION OF THE RMT}

Efforts carried out in the construction and automation of the RMT can be divided into three areas: Mechanics, Computing and Electronics. It is important to highlight that our project is based on hardware and software free (Schwartz et al. 2011, 2013). 
TABLE 1

\section{TECHNICAL CHARACTERISTICS}

\begin{tabular}{l}
\hline Rafael Montemayor Telescope \\
\hline Newtonian focus \\
$f=2027 \mathrm{~mm}$ \\
$D=457.2 \mathrm{~mm}$ \\
$f / D=4.43$ \\
C-ring equatorial mount \\
Approximate weight $=1500 \mathrm{~kg}$ \\
Solid borosilicate mirror \\
CCD Camera: FLI Proline PL16803 monochrome \\
Angular size of the field of view $=60^{\prime} \times 60^{\prime}$
\end{tabular}

${ }^{a}$ FLI: Finger Lakes Instrumentation (http://www . flicamera.com).

\subsection{Electronics}

To move the telescope with help of motors, we used two stepper motors to drive the movements in declination and hour angle, which are coupled to an endless screw and a gear wheel. The stepper motors are controlled by standard drivers (Leadshine DM542 ${ }^{7}$ ). The choice of stepper motors for tracking and pointing was very appropriate, since the telescope manages to reach any position in less than 60 seconds and the tracking movement is continuous and free of vibrations. The speed ramps make the telescope accelerates without the stepper motors losing steps.

We also implemented a safety circuit to avoid movements that can damage the mount. The scripts of this system can be found at https://github.com/telescopio-montemayor/ stepper-hard-limit. To achieve a pointing with a precision lower than 3', we used two 842E Ethernet/IP absolute optical encoders of 18 bits which are directly mounted on the axes of declination and hour angle. In order to interact with the encoders we have developed a series of applications, a server that shows the read of the encoders by means of an api rest and a websocket and a script that synchronizes the mount with them through INDI $^{8}$. We developed the control system based on the project OnStep ${ }^{9}$, which is currently running on an Arduino Mega board. The main reasons for this choice compared to other existing developments are the native support for the correction of the base of

\footnotetext{
${ }^{7}$ Driver Microstep Digital (18-80 Vca - 7.2A peak).

${ }^{8}$ INDI: Instrument-Neutral Distributed Interface (http:// www.clearskyinstitute.com/INDI/INDI.pdf).

${ }^{9}$ OnStep Telescope Controller (https://github.com/ hjd1964/OnStep).
}

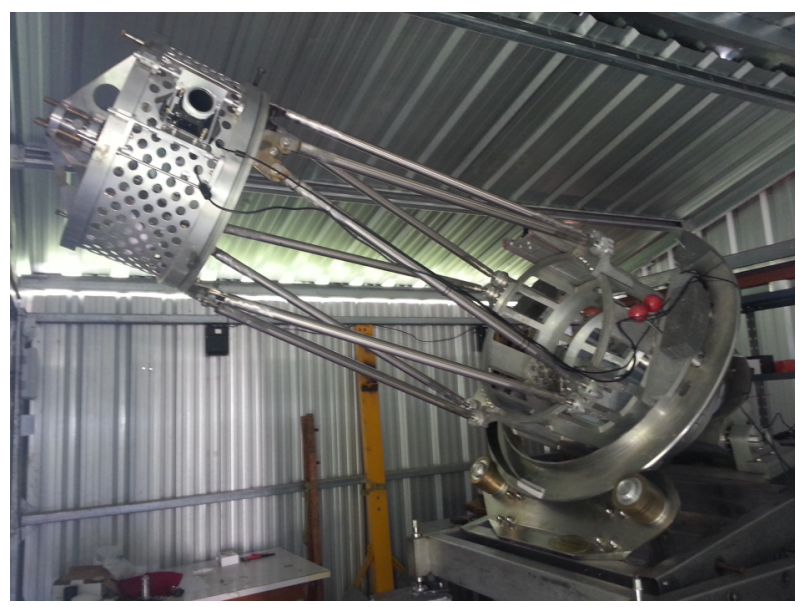

Fig. 1. Rafael Montemayor Telescope at the Faculty of Astronomical and Geophysical Sciences (National University of La Plata, Argentina).

time using the PPS (Pulse Per Second) output of an external GPS unit, the organized and verbose source code and the widely distributed protocol $\left(\mathrm{LX} 200^{10}\right)$, which allows us, if necessary, to use other software apart from INDI, without any problem.

\subsection{Software}

To design the software that allows the remote use of the RMT, we took into account that the telescope will be used for scientific, educational and outreach studies. Based on these requirements, we developed a very versatile web client that can be operated with different permissions of use, called "Rastrosoft" (Boette A. 2017). This software allows the communication in real time among different users. Rastrosoft allows operation of the telescope components through a web page. It also allows to manage observing runs considering different user permissions for the use of the system. By means of this software it is possible to automate image capture sequences, display the image frames in real time for different users in a simultaneous way and communicate among the users through an interactive chat. Rastrosoft supports components that use the INDI standard for communication.

\subsection{Mechanics}

The Mechanical Department of the Faculty made the design and manufacture of the dome, which consists of a rectangular structure of zinc sheet with space to house the telescope and a bench-mounted spectrograph next to the telescope and connected

\footnotetext{
${ }^{10}$ https://www.meade.com/support/TelescopeProtocol_ 2010-10.pdf.
} 
with it by fiber cables. The roof moves laterally, driven by a double motor system, which allows the telescope to access any celestial position with an altitude greater than $30^{\circ}$ above the horizon. As the final location site chosen for the RMT has a highly collimated wind dynamics, the sliding gable roof of the dome will serve as a wind deflector.

\section{FUTURE PROSPECTS}

By the end of 2018 the RMT and its observatory house will be completely dismantled and moved to the province of Salta, Argentina, to be installed at a height of $4800 \mathrm{~m}$ above sea level. The telescope will be used remotely and automatically for observation of exoplanetary transits, search for supernovas, detection of optical sources associated with gravitational wave events and also for astronomical teaching.

\section{REFERENCES}

Boette A. 2017, Tesina de Licenciatura en Sistemas: "Rastro: Sistema web para la utilización de un telescopio remoto", Facultad de Informática, Universidad Nacional de La Plata, Argentina.

Schwartz, M. A., Mauas, P., Melita, M., Buccino, A. P., Pellizza, L., et al. 2011, BAAA, 54, 423

Schwartz, M. A., Perna, P., Mauas, P., Veltri, A., \& Hereñú, H. 2013, BAAA, 56, 449 